\title{
Dilatación de la raíz aórtica en pacientes con válvula bicúspide tras la sustitución valvular aórtica
}

\author{
Víctor Dayan, Leandro Cura, Nelson Pizzano, León Muñoz, Virginia Michelis, Enrique Domínguez, \\ Gerardo Soca, Alejandro Ferreiro.
}

Introducción : La válvula aórtica bicúspide (VAB) está vinculada con la patología aórtica. Pacientes con $\mathrm{VAB}$ tienen elevada probabilidad de requerir cirugía debido a dilatación aórtica o afectación valvular. La progresión en la dilatación aortica luego de la sustitución de la válvula aórtica (SVA) no se ha evaluado en forma definitoria.

Métodos: Un total de 23 pacientes con VAB y diámetro de la raíz aortica inferior a $45 \mathrm{~mm}$ fueron seguidos durante una media de 8 años luego de la SVA. La medición ecocardiográfica de la raíz aórtica y la aorta tubular se realizó a los 6 y 8 años de la cirugía. Se determinaron predictores de dilatación.

Resultados: La raíz aórtica entre 6 y 8 años de seguimiento incrementó significativamente de 34,6 $\pm 7,4$ a $37,6 \pm 7,7 \mathrm{~mm}(\mathrm{p}<0,023)$, mientras que no se encon- traron diferencias con la aorta tubular. La dilatación se observa principalmente en los fumadores, aquellos con antecedentes familiares y en los pacientes dislipémicos. Los factores predictores para dilatación de la raíz aórtica fueron: antecedentes familiares de VAB o de patología aórtica, el tamaño de la prótesis y la superficie corporal (SC). El análisis de regresión multivariado evidenció solo la SC y antecedentes familiares como predictores significativos de dilatación.

Conclusión - La raíz aórtica continúa dilatándose tras la SVA en pacientes con VAB. La SC y la historia familiar de patología aórtica son fuertes predictores de dilatación de la raíz aórtica. Estos pacientes deben ser seguidos de cerca luego de la SVA.

Palabras clave: raíz aórtica, válvula aórtica bicúspide, aorta. 


\section{Aortic root dilatation in patients with Bicuspid aortic valve late after aortic valve replacement}

Background: The presence of a bicuspid aorotic valve $(\mathrm{BAV})$ is associated to aortic disease. Along their lifetime, BAV patients are highly likely to require surgery. Progression of aortic dilatation after aortic valve replacement (AVR) has not been definitely assessed. Methods: A total of $23 \mathrm{BAV}$ patients with aortic diameter $\leq 45 \mathrm{~mm}$ were followed for a mean of 8 years after AVR. The aortic root and proximal ascending aorta were measured by echocardiografy 6 and again 8 years after surgery. Predictors for dilatation were estimated based on demographic data and pharmacologic treatment.

Results: The aortic root diameter increased from $34.6 \pm 7.4$ to $37.6 \pm 7.7 \mathrm{~mm}$ at 6 and 8 years post surgery, respectively ( $\mathrm{p}=0.023$ ). No change was observed at the

\section{Introducción}

La válvula aórtica bicúspide representa la cardiopatía congénita más frecuente con una incidencia del 1-2\% ${ }^{1}$. Se ha visto que estos pacientes tienen un riesgo del 22-25\% de someterse a una intervención quirúrgica en la válvula aórtica o la aorta ascendente ${ }^{2}$. Existe un creciente interés en la relación entre la VAB y la patología de la aorta ascendente ${ }^{3}$.

Se han formulado dos hipótesis para explicar la relación entre la VAB y la patología de la aorta ascendente: la genética y la hemodinámica. Los defensores de la teoría genética sostienen que los pacientes con VAB tiene un defecto intrínseco en la pared aórtica que explica su tendencia a dilatarse así como la agrupación familiar del defecto ${ }^{4,5}$. Los partidarios de la teoría hemodinámica dan importancia al efecto del flujo turbulento a través de la VAB como responsable de la dilatación asimétrica de la aorta ascendente $^{6,7}$. Con el fin de aumentar o disminuir el apoyo a la teoría hemodinámica interesa evaluar la evolución del diámetro de la aorta después de la SVA.

Recientemente, se ha demostrado que en pacientes con dilatación aórtica y estenosis valvular aórtica (tricúspide), tubular aorta . Mean progression of aortic root and tubular aorta during the 2-year interval was $2.9 \pm 4.7 \mathrm{~mm}$ and $0.4 \pm 5.8 \mathrm{~mm}$ respectively. Dilatation was seen mainly in smokers, those with a family history or dyslipidemia. Univariate predictors for aortic root dilatation were: family history of BAV or aortic pathology, prosthesis size and body surface area (BSA). Multivariate regression evidenced only BSA (beta coefficient 11.5) and family history (beta coefficient 4.5) as significant predictors. Conclusion: Aortic root continues to dilate after AVR in BAV patients. Higher BSA and family history of aortic pathology were found to be strong predictors of aortic root dilatation. These patients should be closely followed after AVR.

Keywords: Aortic root; Aortic valve; Aorta la sustitución valvular aórtica (SVA) aislada es suficiente para prevenir la expansión de la aorta a largo plazo ${ }^{8}$. No ha sido evaluado un escenario similar en pacientes con VAB. La elaboración de un estudio destinado a abordar esta cuestión será capaz de ayudar a entender la etiología de la dilatación aórtica en pacientes con VAB luego de la SVA. Hemos demostrado previamente que el diámetro de la raíz aórtica después de 6 años de la SVA en pacientes con VAB no varía significativamente con respecto a su diámetro preoperatorio ${ }^{9}$. Estos resultados se vieron afectados negativamente por el efecto de la aortorrafia, la cual reduce el tamaño de la raíz aórtica y por lo tanto, subestima la comparación entre las dimensiones de raíz aórtica entre el preoperatorio y postoperatorio. Con el fin de neutralizar este efecto, se evaluó el aumento de la raíz aórtica y aorta tubular entre los 6 y los 8 años después de la SVA.

\section{Materiales y Métodos}

Pacientes. A través de nuestra base de datos se identificaron todos los pacientes con VAB que se sometieron a SVA por estenosis y/o regurgitación desde febrero del 2000 a marzo del 2003. Los criterios de 
exclusión fueron reparación concomitante de la aorta ascendente o su reemplazo, diámetro de la raíz aórtica $>45$ mmen el momento de la cirugía (ya que estos pacientes son sometidos a sustitución de la aorta en nuestra institución), endocarditis aguda, evento aórtico agudo, y aquellos con enfermedad del tejido conectivo. Se excluyeron aquellos pacientes perdidos durante el seguimiento o que no pudieron asistir a la evaluación ecocardiográfica. Los pacientes se evaluaron a los 6 y 8 años mediante ecocardiograma, entrevista telefónica y análisis de las historias clínicas. De las variables registradas, la historia familiar se definió como la presencia de válvula aórtica bicúspide o aneurisma de la aorta ascendente en familiares de primer grado. Este estudio fue aprobado por el Comité de Ética del Instituto Nacional de Cirugía Cardíaca y se recabó consentimiento por el paciente.

Procedimiento Quirúrgico: La cirugía se llevó a cabo en la División de Cirugía del Instituto Nacional de Cirugía Cardíaca (INCC). La presencia de VAB y su tipo se confirmó durante la exploración quirúrgica. Se realizó la SVA mediante la técnica estándar ${ }^{10}$ utilizando ya sea prótesis valvular mecánica o biológica.

Ecocardiografía: Se utilizó un ecógrafo Philips Sonos $2000 ®$ con una sonda de 2,0 MHz. Todos los estudios postoperatorios se realizaron de forma prospectiva a los 6 y 8 años de la cirugía y se grabaron en cinta de video para el análisis. En cada paciente, las imágenes fueron adquiridas en un corte longitudinal en una vista paraesternal izquierda. Las mediciones se realizaron perpendicular al eje largo de la aorta, durante la sístole. Los segmentos medidos fueron anillo aórtico, la raíz aórtica y la aorta proximal ascendente (AA) o aorta tubular a un $1 \mathrm{~cm}$ por encima de la unión sino-tubular. La raíz de la aorta (la porción sinusal) se definió como la porción de la aorta entre el anillo aórtico y la unión sino-tubular; aorta tubular se refiere al sector distal a la unión sino-tubular de la AA. Todas las mediciones se realizaron por triplicado y la media fue utilizado para su análisis.

Análisis estadístico. Las variables continuas se expresaron como media \pm desviación estándar (DE). Las variables categóricas se resumieron como porcentaje o como cuentas. Los diámetros de la aorta a los 6 y 8 años de seguimiento se compararon mediante test de t pareado previa valoración de normalidad. Se usó regresión lineal múltiple incluyendo variables que en el análisis univariado demostraron tener una significación $<0,1$. Los valores de $\mathrm{p}<0,05$ fueron considerados significativos.

\section{Resultados}

Un total de 23 pacientes con VAB se incluyeron en el estudio. La orientación de los velos fue anteroposterior en 22 de los 23 pacientes (96\%). Los datos demográficos así como diámetros aórticos se muestran en la Tabla 1. El diámetro preoperatorio de la raíz aórtica fue de 36,6 \pm $4,6 \mathrm{~mm}$. Luego de una media de 6 y 8 años de la SVA, el diámetro de la raíz aórtica fue de $34,6 \pm 7,4 \mathrm{~mm}$ y $37,6 \pm$ $7,7 \mathrm{~mm}$, respectivamente $(\mathrm{p}<0,023)$. El diámetro de la aorta tubular a los 6 y 8 años de seguimiento fue de 37,8 $\pm 5,8 \mathrm{~mm}$ y $38,2 \pm 7,1 \mathrm{~mm}$, respectivamente (NS). $\mathrm{La}$ dilatación de la raíz aórtica y aorta tubular entre los 6 y 8 años de seguimiento fue de $2,9 \pm 4,7 \mathrm{~mm}$ y $0,4 \pm 5,8 \mathrm{~mm}$, respectivamente.

\begin{tabular}{|l|l|}
\hline $\begin{array}{c}\text { Tabla 1: Características demográficas y diámetro aórtico en } \\
\text { pacientes con VAB (n=23) }\end{array}$ \\
\hline Edad (años) & $60 \pm 11$ \\
Masculino & $52 \%$ \\
Tabaquismo & $56 \%$ \\
Hipertensión & $74 \%$ \\
Diabetes & $26 \%$ \\
Dislipidemia & $61 \%$ \\
Estenosis & $56 \%$ \\
SC (m2) & $1.83 \pm 0.22$ \\
Beta bloqueantes & $17 \%$ \\
Estatinas & $35 \%$ \\
Historia familiar & $30 \%$ \\
VAB de orientación antero-posterior & $96 \%$ \\
Prótesis mecánica & $73 \%$ \\
Diámetro protésico (mm) & $23 \pm 3$ \\
Diámetro basal de raíz (mm) & $36.6 \pm 4.6$ \\
Diámetro de ráz a 6 años (mm) & $34.6 \pm 7.4$ \\
Diámetro de raíz a 8 años (mm) & $37.6 \pm 7.7$ \\
Diámetro tubular a 6 años (mm) & $37.8 \pm 5.8$ \\
Diámetro tubular a 8 años (mm) & $38.2 \pm 7.1$ \\
Dilatación de raíz (mm) & $2.9 \pm 4.7$ \\
Dilatación tubular (mm) & $0.4 \pm 5.8$ \\
\hline
\end{tabular}

SC: Superficie corpórea. VAB: Válvula aórtica bicúspide.

Evolución del diámetro aórtico entre los 6 y 8 años de seguimiento después de la SVA. La dilatación de la raíz aórtica durante el período de estudio fue significativa en pacientes con antecedentes familiares, tabaquistas 
y dislipidémicos. Aquellos pacientes tratados con betabloqueantes y estatinas no presentaron aumento del diámetro de la raíz aórtica durante dicho período (Tabla 2). El análisis univariado mostró como predictores de la dilatación de la raíz aórtica la presencia de antecedentes familiares, área de superficie corporal y el tamaño de la prótesis (Tabla 3). Después de incluir estas variables en el modelo de regresión multivariado, surgió un modelo significativo $(F=12.38, p<0,01)$ con un $R$ al cuadrado $=0,553$. El área de superficie corporal y los antecedentes familiares fueron los únicos predictores significativos para la dilatación de la raíz aórtica con un coeficiente beta de 11,5 y 4,5 , respectivamente (Tabla 4).

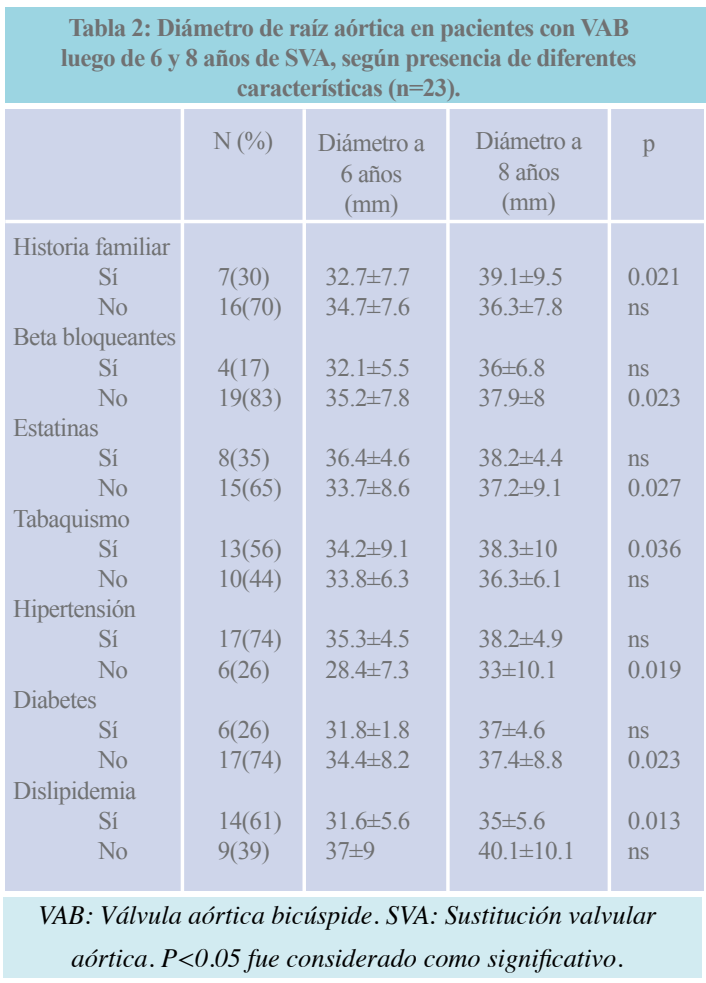

\begin{tabular}{|c|c|c|}
\hline \multicolumn{3}{|c|}{$\begin{array}{c}\text { Tabla } 3 \\
\begin{array}{c}\text { Análisis univariado para predictores de dilatación de raíz } \\
\text { aórtica }(\mathrm{n}=23) \text {. }\end{array}\end{array}$} \\
\hline Variables & Coeficiente Beta & $\mathrm{p}$ \\
\hline $\begin{array}{l}\mathrm{SC} \\
\text { Diámetro protésico } \\
\text { Historia familiar }\end{array}$ & $\begin{array}{l}12.57 \pm 3.71 \\
0.77 \pm 0.36 \\
5.10 \pm 1.88\end{array}$ & $\begin{array}{l}0.003 \\
0.044 \\
0.013\end{array}$ \\
\hline
\end{tabular}

Análisis de regresión lineal entre la dilatación aórtica. SC: Superficie corporal. $P<0.05$ fue considerado como significativo.

\begin{tabular}{|l|l|l|} 
Tabla 4. \\
\begin{tabular}{|l|l|} 
Análisis de regresión lineal múltiple para predictores de \\
dilatación aórtica entre $\mathbf{6}$ y 8 años luego de la SVA
\end{tabular} \\
\hline Predictor & Coeficiente B & $\mathrm{p}$ \\
\hline $\begin{array}{l}\text { Historia familiar } \\
\text { Superficie corporal }\end{array}$ & $\begin{array}{l}4.49 \pm 1.50 \\
11.53 \pm 3.17\end{array}$ & 0.01 \\
\hline
\end{tabular}

\section{Discusión}

La VAB representa la cardiopatía congénita más frecuente del adulto. Su relación con la aortopatía ha sido objeto de intensa investigación ${ }^{15-20}$. Las guías de recomendación actuales sugieren la sustitución de la aorta ascendente en pacientes con VAB cuando su diámetro sea superior a 50 $\mathrm{mm}^{11,12}$ en comparación con los pacientes con válvula tricúspide en el que su diámetro debe superar los $55 \mathrm{~mm}$. Estas recomendaciones están apoyadas en dos grandes estudios de seguimiento de los pacientes con $\mathrm{VAB}^{2,13}$. Éstos demuestran que aproximadamente el $25 \%$ de estos pacientes en algún momento de su vida sufren eventos clínicos relacionados a patología valvular aórtica o requieren cirugía de aorta ascendente ${ }^{2,13}$. Borger et al., demostraron que pacientes con VAB y diámetro aórtico $>45 \mathrm{~mm}$ en los cuales tan sólo se realiza SVA, experimentan menor supervivencia y mayor incidencia de eventos aórticos agudos $^{14}$. Por lo tanto, ¿cuál es la explicación para el aumento de la patología aórtica en pacientes con VAB? Se proponen dos teorías no-excluyente : las teorías genética y la hemodinámica.

La teoría genética postula un defecto intrínseco en la pared de la aorta como la principal causa de la aortopatía ${ }^{5,15,16}$. Esta teoría está apoyada principalmente por el hallazgo de dilatación en el autoinjerto pulmonar de los pacientes con VAB que se someten a procedimiento de Ross en comparación con los pacientes portadores de válvula aórtica tricúspide ${ }^{16 .}$ Este hallazgo permite suponer que los pacientes con VAB tienen alteraciones vasculares que no se limitan a la aorta. Las alteraciones vasculares intrínsecas son hereditarias y ayudan a explicar el agrupamiento familiar del defecto, así como la asociación entre pacientes con VAB y familiares de primer grado con aortopatía y válvula tricúspide ${ }^{4,17}$.

Los seguidores de la teoría hemodinámica proponen que aunque la VAB no sea estenótica ni regurgitante, hay un flujo turbulento que es responsable de "shear stress" en la 
pared aórtica y predispone a su dilatación asimétrica ${ }^{6,7,18}$. Un informe reciente describe una deficiencia en proteínas de matriz extracelular y expresión de óxido nítrico en el segmento aórtico enfrentado a la región de máximo flujo turbulento y dilatación ${ }^{19}$.

Con el fin de contribuir a aclarar esta cuestión, consideramos de gran utilidad evaluar la progresión de la raíz aórtica y aorta tubular después de la SVA. De esta manera, podríamos seguir la dimensión aórtica sin la turbulencia generada por la VAB. Recientemente se ha publicado un estudio similar pero en pacientes con válvula tricúspide ${ }^{8}$. Estos autores concluyeron que la SVA fue capaz de evitar la dilatación de la aorta ascendente. Nuestro grupo ha demostrado previamente ausencia en la dilatación de la raíz aórtica a los 6 años de seguimiento cuando se compara con el diámetro preoperatorio ${ }^{9}$. No obstante, la aortorrafia genera un efecto reductor en la aorta ascendente que probablemente subestima la diferencia en diámetro al comparar el diámetro preoperatorio con aquel a los 6 años de seguimiento.

Nuestros resultados, muestran que a pesar de que no hay una dilatación significativa a los 8 años de seguimiento en comparación con las dimensiones preoperatorias (probablemente debido al efecto de la aortorrafia), la raíz aórtica se dilata de manera significativa entre los 6 y 8 años después de la SVA, sin dilatación significativa de la aorta tubular.

No hay consenso en cuanto a qué porción de la aorta ascendente sufre mayor dilatación en pacientes con VAB. Schaefer et $\mathrm{al}^{6}$ sugieren que el fenotipo de VAB (si es antero-posterior o lateral) influye en el segmento aórtico susceptible a la dilatación. La VAB con una orientación anterior-posterior de la válvula (fenotipo más frecuente) dilatará la raíz aórtica, mientras que la orientación derecha-izquierda de la válvula, dilata la aorta tubular ${ }^{6}$. A pesar de que nuestros hallazgos apoyan la teoría genética, no podemos excluir la posibilidad de que el shear stress inicial, que afecta principalmente a la raíz aórtica debido a la configuración anterior-posterior, se traduzca en una alteración de las características moleculares de la pared de la raíz aórtica, que termina contribuyendo a su dilatación, incluso después de SVA ${ }^{19}$. En nuestra población estudiada, el $96 \%$ tenían la orientación antero-posterior y esto podría ser la causa de la dilatación preferente de la raíz aórtica, sin embargo, no estamos en condiciones de afirmarlo de manera concluyente debido al bajo número de pacientes incluidos.
La dilatación de la raíz aórtica fue de aproximadamente $3 \mathrm{~mm}$. Utilizando análisis de regresión simple, sólo la historia familiar, el tamaño de la prótesis y la superficie corporal fueron predictores significativos. Los antecedentes familiares y el tabaquismo ya se han asociado con dilatación aórtica en pacientes con VAB previa a la SVA, pero no se ha reportado su asociación después de la SVA ${ }^{20,4}$.

No encontramos ninguna publicación acerca de la asociación de dilatación de la raíz aórtica con dislipemia, la cual podría explicarse debido a la disfunción endotelial que caracteriza a los pacientes dislipémicos ${ }^{21}$. Las estatinas han sido estudiadas por su efecto en detener la progresión del aneurisma de aorta abdominal ${ }^{21}$. Ningún estudio ha evaluado su papel en la prevención de la dilatación aórtica en pacientes con VAB luego de la SVA. El uso de beta bloqueantes ha sido recomendado para disminuir la progresión de la dilatación de la aorta ascendente en pacientes con síndrome de Marfán ${ }^{22}$. Hemos encontrado que los pacientes tratados con beta-bloqueantes no presentaron dilatación significativa. Ésto puede explicarse, al igual que con las estatinas, por la función protectora para la disfunción endotelial que se ha atribuido a los beta bloquean$\operatorname{tes}^{23}$. Estos resultados deben tomarse con cautela, ya que el número de pacientes en la muestra es pequeño como para sacar conclusiones sólidas. Sorprendentemente, no se evidenció dilatación significativa en pacientes hipertensos y diabéticos, lo cual es debido probablemente a que la mayoría recibían beta bloqueantes como tratamiento antihipertensivo. Sin embargo, el pequeño número de pacientes en ambos subgrupos podría ser la razón para no tener diferencias estadísticas.

Tras el análisis multivariado, sólo encontramos la SC y los antecedentes familiares como predictores de dilatación de la raíz aórtica. Por cada incremento de $0,2 \mathrm{~m}^{2}$ de superficie corpórea, la raíz aórtica aumentó aproximadamente 1 mm por año después de la cirugía. De acuerdo con nuestro modelo, la presencia de historia familiar aumenta la tasa de dilatación anual de la raíz aórtica en $2 \mathrm{~mm}$. La influencia de la historia de la familia incluso podría estar subestimada dado que no se le realizó ecocardiograma a ningún miembro de la familia.

En trabajos anteriores se ha descrito que después de la SVA en pacientes con VAB se dilata el segmento tubular y no la raíz de la aorta ${ }^{24}$. La explicación de la diferencia con nuestros hallazgos se debe probablemente al hecho de que los autores compararon el diámetro de la raíz aórtica en el seguimiento con el preoperatorio. Por lo tanto sus 
comparaciones probablemente están subestimadas debido al efecto de la aortorrafia sobre la raíz aórtica.

Nuestros resultados ayudan a predecir qué pacientes están en mayor riesgo de sufrir dilatación de la raíz aórtica así como el tiempo estimado para alcanzar dimensiones riesgosas de ruptura. De esta manera podemos identificar la población de pacientes con VAB que podrían beneficiarse tanto de una estrategia de sustitución aórtica profiláctica como de seguimiento cercano, principalmente aquellos pacientes jóvenes (en los que su esperanza de vida es mucho mayor que el tiempo estimado de la raíz aórtica para llegar a $45 \mathrm{~mm}$ ), con elevada SC y antecedentes familiares de VAB y/o aortopatía.

Aunque nuestros resultados no proporcionan una respuesta definitiva con respecto a la patogénesis de la dilatación aórtica en pacientes con VAB, apoyan en parte la teoría genética. Sin embargo no estamos en condiciones de afirmar que la SVA no detiene la dilatación de la raíz aórtica. Tal vez, si en estos pacientes no se hubiera realizado la SVA la aorta se hubiese dilatado mucho más rápido.

Serán necesarios estudios poblacionales más grandes para definir la indicación individualizada para la sustitución de la raíz aórtica basada en la presencia de los factores de riesgo antes mencionados. Aunque el número de pacientes evaluados es pequeño, estos resultados deben ser tenidos en cuenta en la práctica clínica y ser la base estudios clínicos de mayor envergadura.

Limitaciones. La principal limitación de nuestro estudio esel pequeño número de pacientes. Su diseño retrospectivo es un importante inconveniente que es difícil de resolver debido a razones éticas y otra limitación importante es no tener los diámetros de la aorta inmediatamente después de la cirugía con el fin de hacer comparaciones a intervalos más largos.

\section{Referencias:}

1. TADROS TM, KLEIN MD, SHAPIRA OM. Ascending aortic dilatation associated with bicuspid aortic valve. Pathophysiology, molecular biology, and clinical implications. Circulation 2009;119:880-890.

2. TZEMOS N, THERRIEN J, YIP J, THANASSOULIS G, TREMBLAY S, JAMORSKI MT, et al. Outcomes in adults with bicuspid aortic valves. JAMA 2008;300:1317-325

3. GIRDAUSKAS E, BORGER MA, SECKNUS MA, GIRDAUSKAS G, KUNTZE T. Is aortopathy in bicuspid aortic valve disease a congenital defect or a result of abnormal hemodynamics? A critical reappraisal of a one-sided argument. Eur J Cardiothorac Surg 2011; 39: 809-14.

4. BINER S, RAFIQUE AM, RAY I, CUK O, SIEGEL RJ, TOLSTRUP K. Aortopathy is prevalent in relatives of bicuspid aortic valve patients. J Am Coll Cardiol 2009; 53:
2288-95.

5. NISTRI S, GRANDE-ALLEN J, NOALE M, BASSO C, SIVIERO P, MAGGI S, et al. Aortic elasticity and size in bicuspid aortic valve syndrome. Eur Heart J. 2008; 29 : 472-9.

6. SCHAEFER BM, LEWIN MB, STOUT KK, BYERS PH, OTTO CM. Usefulness of bicuspid aortic valve phenotype to predict elastic properties of the ascending aorta. Am J Cardiol. 2007; 99: 686-90

7. ESPERANZA MD, TA HOPE, AK MEADOWS, KG ORDOVAS, TH URBANIA, CALLEJÓN MT, et al. La válvula aórtica bicúspide: cuatro dimensiones de evaluación RM de aorta ascendente patrones de flujo sistólico. Radiología. 2010; 255:53-61.

8. GAUDINO M, ANSELMI A, MORELLI M, PRAGLIOLA C, TSIOPOULOS V, GLIECA F, et al. Aortic expan- 
sion rate in patients with dilated post-stenotic ascending aorta submitted only to aortic valve replacement long-term follow-up. J Am Coll Cardiol. 2011;58:581-4.

9. DAYAN V, CURA L, MUÑOZ L, ARECO D, FERREIRO A, PIZZANO N. Risk of subsequent aortic dilatation is low in patients with bicuspid aortic valve and normal aortic root diameter at the time of aortic valve replacement. Interact Cardiovasc Thorac Surg. 2010;10:535-8.

10. CZER LS, CHAUX A, MATLOFF JM, DEROBERTIS MA, NESSIM SA, SCARLATA D, et al. Ten-year experience with the St. Jude medical valve for primary valve replacement. J Thorac Cardiovasc Surg 1990; 100: 44-54.

11. BONOW RO, CARABELLO BA, CHATTERJEE K, DE LEON AC JR, FAXON DP, FREED MD, et al. 2008 focused update incorporated into the ACC/AHA 2006 guidelines for the management of patients with valvular heart disease. J Am Coll Cardiol. 2008;52:e1-142.

12. HIRATZKA LF, BAKRIS GL, BECKMAN JA, BERSIN RM, CARR VF, CASEY DE JR, et al. Guidelines for the diagnosis and management of patients with thoracic aortic disease. J Am Coll Cardiol. 2010;55:e27-e129.

13. MICHELENA HI, DESJARDINS VA, AVIERINOS JF, RUSSO A, NKOMO VT, SUNDT TM, et al. Natural history of asymptomatic patients with normally functioning or minimally dysfunctional bicuspid aortic valve in the community. Circulation 2008;117:2776-84.

14. BORGER MA, PRESTON M, IVANOV J, FEDAK PW, DAVIERWALA P, ARMSTRONG S, et al. Should the ascending aorta be replaced more frequently in patients with bicuspid aortic valve disease? J Thorac Cardiovasc Surg. 2004;128:677-83.

15. DE SA M, MOSHKOVITZ Y, BUTANY J, DAVID TE. Histologic abnormalities of the ascending aorta and pulmonary trunk in patients with bicuspid aortic valve disease: clinical relevance to the ross procedure. J Thorac Cardiovasc Surg. 1999;118:588-94.

16. DAVID TE, OMRAN A, IVANOV J, ARMSTRONG S,
DE SA MP, SONNENBERG B, et al. Dilation of the pulmonary autograft after the Ross procedure. J Thorac Cardiovasc Surg. 2000;119:210-20.

17. CRIPE L, ANDELFINGER G, MARTIN LJ, SHOONER $\mathrm{K}$, BENSON DW. Bicuspid aortic valve is heritable. J Am Coll Cardiol. 2004;44:138-43.

18. AICHER D, URBICH C, ZEIHER A, DIMMELER S, SCHÄFERS HJ. Endothelial nitric oxide synthase in bicuspid aortic valve disease. Ann Thorac Surg. 2007;83:1290-4

19. COTRUFO M, DELLA CORTE A, DE SANTO LS, QUARTO C, DE FEO M, ROMANO G, et al. Different patterns of extracellular matrix protein expression in the convexity and the concavity of the dilated aorta with bicuspid aortic valve: preliminary results. J Thorac Cardiovasc Surg. 2005;130:504-11.

20. IQTIDAR AF, O'ROURKE DJ, SILVERMAN DI, THOMPSON PD, DISCIPIO AW, PALAC RT. Predictors of rapid aortic dilatation in adults with a bicuspid aortic valve. J Heart Valve Dis. 2011;20:292-8.

21. MIYAKE T, MORISHITA R. Pharmacological treatment of abdominal aortic aneurysm. Cardiovasc Res. 2009;83:436-43.

22. SHORES J, BERGER KR, MURPHY EA, PYERITZ RE. Progression of aortic dilatation and the benefit of long-term beta-adrenergic blockade in Marfan's syndrome. N Engl J Med. 1994;330:1335- 41.

23. PUDDU P, PUDDU GM, CRAVERO E, MUSCARI A. Different effects of antihypertensive drugs on endothelial dysfunction. Acta Cardiol. 2004;59:555-64.

24. YASUDA H, NAKATANI S, STUGAARD M, TSUJITAKURODA Y, BANDO K, KOBAYASHI J, et al. Failure to prevent progressive dilation of ascending aorta by aortic valve replacement in patients with bicuspid aortic valve: comparison with tricuspid aortic valve. Circulation. 2003;108:II291-4. 